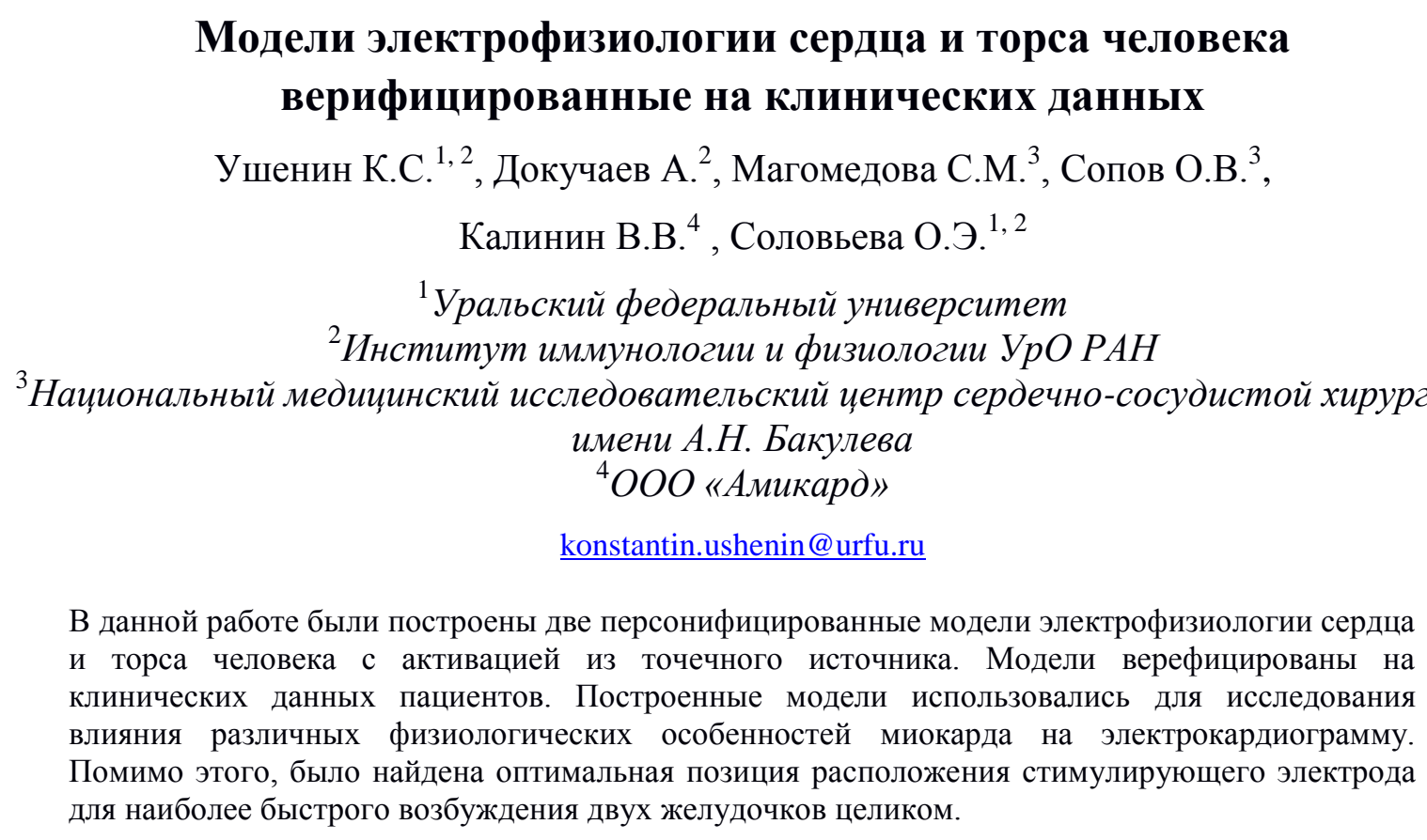

Ключевые слова: электрофизиология миокрада, бидоменная модель, метод конечных элементов, персонализированное моделирование.

\title{
Models of human heart and torso electrophysiology verified against clinical data
}

\author{
Ushenin K.S. ${ }^{1,2}$, Dokuchaev A. ${ }^{2}$, Magomedova S.M. ${ }^{4}$, Sopov O.V. ${ }^{4}$, Kalinin V.V. ${ }^{4}$, Solovyova O. ${ }^{1,2}$ \\ ${ }^{1}$ Ural Federal University \\ ${ }^{2}$ Institute of Immunology and Physiology Ural Branch of RAS \\ ${ }^{3}$ Bakoulev Scientific Center for Cardiovascular Surgery \\ ${ }^{4}$ OOO Amikard
}

\begin{abstract}
We present two models of the human heart and the torso electrophysiology with activation from one point. The models are verified against clinical data. Effects of the various physiological properties are studied within the models. Also, we determine the optimal position of the stimulation electrode tip location that provided the fastest activation of the ventricles.
\end{abstract}

Keywords: myocardium electrophysiology, bidomain model, finite element method, personalized model.

\section{1. Введение}

Математическое моделирование живых систем позволяет проводить эксперименты, которые невозможны в in-vivo или ex-vivo подходах, значительно расширяя круг фундаментальных вопросов на которые может ответить исследователь.

В основе математического моделирования живых систем лежит принцип верефикации моделей относительно биологического или биофизического экспериментов. Однако модели человеческих органов могут быть также верефицированы относительно клинических данных пациентов.

В нашей работе мы построили электрофизиологические модели сердца и торса двух пациентов на основе данных компьютерной томографии (КТ). Далее эти модели были верефицированы с использованием записи электрокардиограммы в 224 отведениях на поверхности торса.

Построенные модели были использованы для исследования влияния физиологических свойств нормального миокарда на электрокардиограмму (ЭКГ) и для определения оптимальной позиции конца стимулирующего элетрода, обеспечивающего наиболее быструю активацию желудочков сердца.

\section{2. Методы}

\section{1. Клинические данные}


Таблица 1. Данные о пациентах

\begin{tabular}{|c|c|c|}
\hline & Случай 1 & Случай 2 \\
\hline Пол & Ж & M \\
\hline Возраст & 67 & 56 \\
\hline $\begin{array}{c}\text { Ширина } \\
\text { QRS }\end{array}$ & 208 & 230 \\
\hline $\begin{array}{c}\text { Основной } \\
\text { диагно3 }\end{array}$ & $\begin{array}{c}\text { Аритмогенная } \\
\text { кардиоммиопатия }\end{array}$ & $\begin{array}{c}\text { Гипертрофическая } \\
\text { кардиомиопатия }\end{array}$ \\
\hline
\end{tabular}

Для исследования были использованы данные двух пациентов с имплантированными устройствами сердечной ресинхронизирующей терапии (СРТ, см. табл. 1). Пациенты прошли компьютерную томографию (КТ) и запись поверхностной электрограммы в 240 отведениях с поверхности торса (Amycard 01C [1]) во время программирования устройства сердечной ресинхронизирующей терапии.

Трехмерная модель торса и сердца построена с использованием системы Amycard 01C (рис. 1). Объемная трехмерная сетка построена с использованием открытого программного обеспечения GMSH [2]. Точное положение конца электрода восстановлено по данным КТ.

\section{2. Модель электрофизиологии}

Для описания электрофизиологии миокарда использовалась бидоменная модель [3]. В качестве модели трансмембанных ионных токов использовалась модель кардиомиоцита желудочка сердца человека [4].

Реалистичное поле направлений волокон в миокарде задавалось методом Байера [5].

Апико-базальная клеточная неоднородность по длительности потенциала действия задавалась методом Келлера [6] при помощи градиента параметров для медленного калиевого тока IKs. Трансмуральная клеточная неоднородность стенках желудочков задавалась в отношении $50 \%$ эпикардиальных клеток, 50 \% эндокардиальных клеток. Емкость мембраны и клеточная неоднородность задавалась согласно [4]. Отношение площади поверхности мембран к объему задавалась путем подбора значений на основании ширины QRS во II отведении.

Для моделирования применялось программное обеспечение с открытым исходным кодом [7].

Апикобазальный градиент длительности потенциала действия составил от 268 мс до 330 мс. Скорость проведения возбуждения вдоль волокон $0.6 \mathrm{~m} / \mathrm{c}$, поперек волокон - $0.2 \mathrm{~m} / \mathrm{c}$. Полученные модельные значения лежат в физиологических диапазонах и соотносятся с правильной шириной QRS комплекса. Пример карты активации и потенциалов на торсе при возбуждении, имитирующем стимуляцию левожелудочковым электродом представлены на рисунке 1.

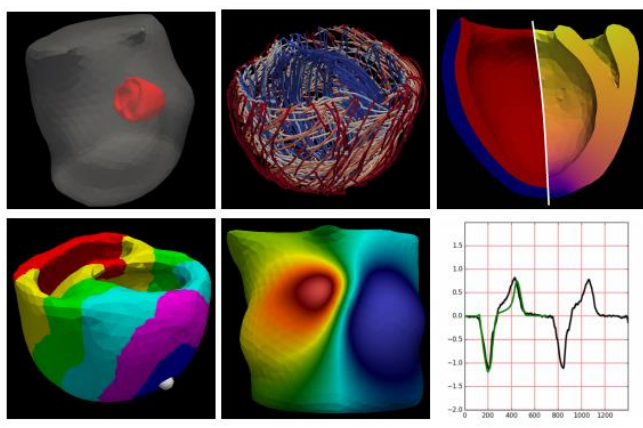

Рис. 1. Слева на право, сверху вниз: анатомическая модель, направление волокон (выборочно), трансмуральная и апикобазальная неоднородности, активационная карта на поверхности сердца, карта потенциалов на поверхности торса, сравнение реальной ЭКГ (черная) и смоделированной (зеленая).

\section{2. Методы верефикации модели}

$$
\begin{gathered}
\mathrm{SC}(x, y)=\frac{\sum_{i=0}^{T} x_{t} y_{t}}{\sqrt{\sum_{i=0}^{T} x_{t} \sum_{i=0}^{T} y_{t}}}, \operatorname{ED}(x, y)=\sqrt{\sum_{i=0}^{T}\left(x_{t}-y_{t}\right)^{2}}, \\
\mathrm{ZM}_{\Delta t, \mu}(x, y)=\min _{\Delta t, \mu} \sqrt{\sum_{t=0}^{T}\left(x_{t}-\operatorname{zoom}\left(y_{t}+\Delta t, \mu\right)\right)^{2}} .
\end{gathered}
$$

Мы использовали три метрики для сравнения модельных и экспериментальных сигналов ЭКГ и анализа результатов. SC - корреляционная метрика, чувствительна к правильной ориентации QRS комплекса и Т волны. ED - евклидово расстояние между сигналами, чувствительно к правильной ориентации волн ЭКГ и амплитудам сигналов. ZM модифицированная ED метрика, учитывающая возможные временные сдвиги и масштабирование по времени (в малом диапазоне значений) модельного сигнала по отношению к экспериментальному.

Скорость проведения по миокарду различается для субъектов, но в модели используются обобщенные значение, полученные на основе популяционных данных. Метрика ZM позволяет не учитывать влияние слабой разницы по скорости проведения между клиническими данными и модельными.

\section{3. Результаты верефикации}

Разработанные модели были верифицированы на основе клинических данных ЭКГ, записанных в 240 отведениях на торсе при стимуляции из ЛЖ электрода. Значения коэффицента корреляции сигналов для более чем $50 \%$ электродов лежат внутри интервала от 0.65 до 0.9 , и более $80 \%$ электродов показывают корреляцию модельных и экспериментальных сигналов более 0.5 (рис. 2 и рис. 3). Данные количественные оценки находятся в пределах величин, ранее полученных при нормальной последовательности активации 
желудочков [6]. Электроды, выходящие за "усы" box-plot диаграммы, в основном локализованы в левой передней части торса. В данном регионе модель показывает инвертированные QRS и Tволну. Тем не менее, мы считаем, что модель в целом хорошо воспроизводит клинические данные и может быть использована для исследований влияния на ЭКГ различных параметров миокарда и местоположения точки стимуляции.
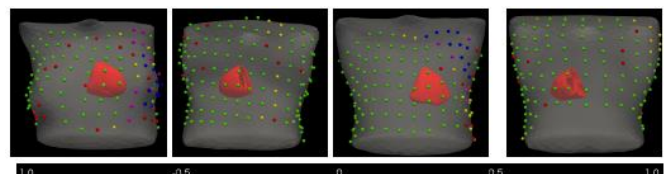

Рис. 2. Значения коллеряционной метрики на отдельных электродах.

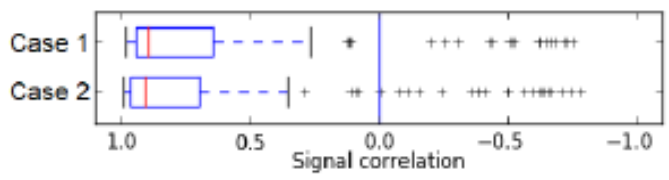

Рис. 3. Распределение корреляции сигналов для каждого случая.

\section{4. Результаты и обсуждение}

Таблица 2. Результаты анализа чувствительности моделей к различным физиологическим параметрам. ED метрика

\begin{tabular}{|c|c|c|c|c|}
\hline & \multicolumn{2}{|c|}{ ED метрика } & \multicolumn{2}{c|}{ ZM метрика } \\
\cline { 2 - 5 } & Сл. 1 & Сл. 2 & Сл. 1 & Сл. 2 \\
\hline $\begin{array}{c}\text { Референсная } \\
\text { модель } \\
\text { (коэффициент }\end{array}$ & वнизотропии 9:1, \\
$\begin{array}{c}\text { апикобазальная } \\
\text { неоднородность, } \\
\text { активация с } \\
\text { субэпикарда) }\end{array}$ & $100 \%$ & $100 \%$ & $100 \%$ & $100 \%$ \\
\hline $\begin{array}{c}\text { Изотропия } \\
\text { (коэффициент } \\
\text { анизотропии } \\
1: 1)\end{array}$ & $140 \%$ & $150 \%$ & $114 \%$ & $116 \%$ \\
\hline $\begin{array}{c}\text { Умеренный } \\
\text { коэффициент } \\
\text { анизотропии } \\
4: 1\end{array}$ & $90 \%$ & $97 \%$ & $88 \%$ & $92 \%$ \\
\hline $\begin{array}{c}\text { Высокая степень } \\
\text { анизотропии } \\
16: 1\end{array}$ & $107 \%$ & $104 \%$ & $105 \%$ & $104 \%$ \\
\hline $\begin{array}{c}\text { Нет } \\
\text { неоднороности }\end{array}$ & $108 \%$ & $106 \%$ & $101 \%$ & $102 \%$ \\
\hline $\begin{array}{c}\text { Варьирование } \\
\text { положение } \\
\text { точки активации }\end{array}$ & $118 \%$ & - & $\begin{array}{c}98 \%- \\
118 \%\end{array}$ & - \\
\hline $\begin{array}{c}\text { Активация с } \\
\text { субэндокарда }\end{array}$ & $107 \%$ & - & $106 \%$ & - \\
\hline
\end{tabular}

\section{1. Влияние анизиотропии миокарда}

Для оценки влияния анизотропии миокарда мы зафиксировали проводимость вдоль волокон и варьировали коэффициент анизотропии вдоль / поперек волокон. Рассмотрено 4 варианта для каждой персонифицированной модели с различным коэффициентом анизотропии: 1:1 (изотропная ткань), 4:1 (умеренная анизотропия), 9:1 (референсная анизотропная модель с широко используемым в моделировании коэффициентом анизотропии) и 16:1 (высокая степень анизотропии).

Обнаружена существенная чувствительность ЭКГ к параметрам анизотропии миокарда. При этом максимальная разница наблюдается при сравнении с изотропным миокардом (табл. 2).

\section{2. Клеточная неоднородность}

Для оценки влияния роли клеточной неоднородности мы исключили ее из модели. При этом в отличие от результатов для нормальной активации миокарда [6] мы не получили инверсии Т-волны и существенных изменений на ЭКГ (табл. 2).

\section{3. Влияние положения точки стимуляции 1}

Проведена оценка влияния смещения стимулирующего электрода на модельные ЭКГ.

Проанализированы результаты модели для 18 случайно расположенных точек стимуляции, смещенных на 2-3 сантиметра от референсной точки (заданной на основе КТ), а так же при перемещении точки стимуляции с субэпикарда на субэндокард. Интерполируя полученные результаты, мы обнаружили, что карта значений ошибки модельной ЭКГ в зависимости от величины смещения электрода относительно референсной позиции является выпуклой функцией с минимумом в диапазоне 1 см от референсной позиции электрода (рис. 4). Кроме этого, мы показали, что перенос точки симуляции с субэпикарда на субэндокард приводит к существенному изменению ЭКГ.

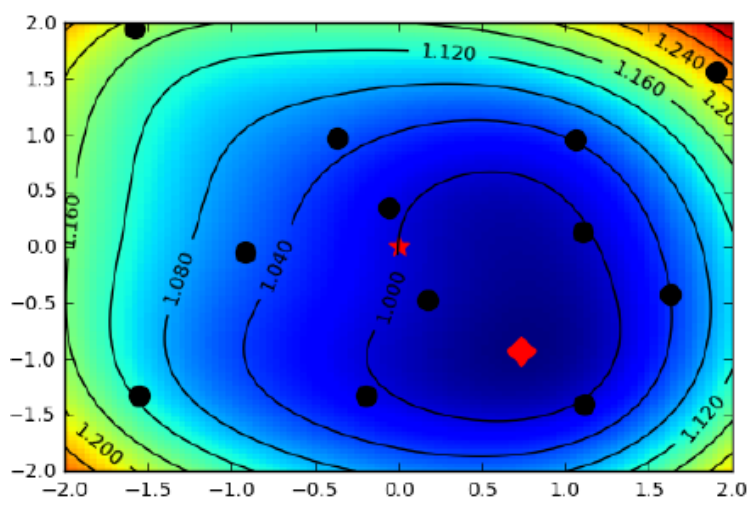

Рис. 4. Карта ошибки между реальными и модельными сигналами ЭКГ при смещении точки активации в модели. Красная звезда - позиция электрода по КТ. Черные точки - случайные позиции электродов. Красный ромб - точка минимума. 


\section{4. Влияние положения точки стимуляции 2}

Исследована связь между временем полной активации желудочков и шириной QRS при точечной активации с субэпикарда из различных сегментов стенки в традиционной 17-сегментной модели разбиения левого желудочка (Bull's eye). Минимальное время активации наблюдалось при стимуляции ЛЖ из 17 сегмента, т.е. с верхушки ЛЖ (рис. 5). При любом положении точки активации в ЛЖ, время активации двух желудочков больше ширины QRS на 40-60 мс (рис. 6).

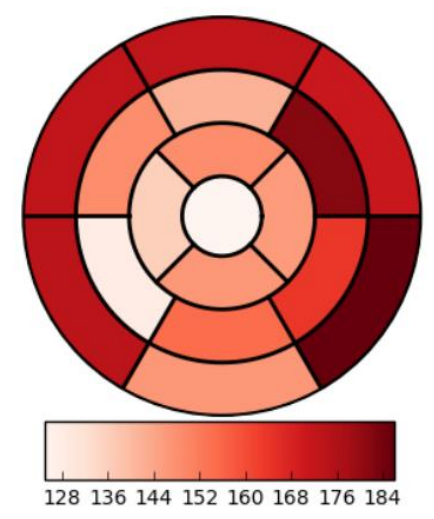

Рис. 5. Длительность QRS комплекса ЭКГ при расположении точки активации в разных сегментах ЛЖ.

\section{5. Заключение}

В нашем исследовании были построены персонализированные модели электрофизиологии сердца и торса человека при условии активации миокарда из одной точки. Верефикация проведена на основе клинических данных.

В ходе исследований мы ранжировали некоторые особенности электрофизиологии миокарда по степени их влияния на ЭКГ. Наибольшее влияние оказывает положение точки стимуляции, затем идет анизотропия миокарда и апико-базальная неоднородность. Наименьшее влияние оказывает трансмуральная неоднородность.

Так же было установлено, что наиболее быстрая активация происходит при активации желудочков сердца из верхушки левого желудочка (17 сегмент Bull's eye).

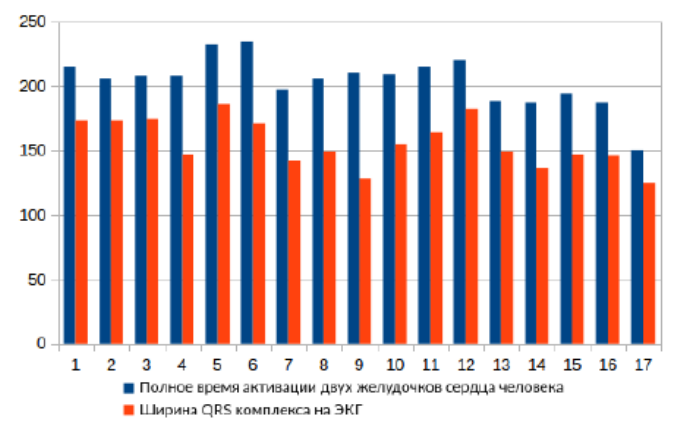

Рис. 6. Связь времени полной активации желудочков и ширины QRS при стимуляции из 17 сегментов ЛЖ.

\section{6. Благодарности}

Работа

поддержана

постановлением Правительства РФ № 211 от 16.03.2013 (соглашение 02.А03.21.0006), РФФИ (научный проект № 18-3100401), Программой Президиума РАН № 27 (тема АААА-А18-118020590030-1), госзаданием ИИФ УрО РАН (тема № АААА-А18-118020590031-8).

Для проведения вычислительных экспериментов использовался суперкомпьютер Уральского федерального университета и суперкомпьютер «УРАН» Института математики и механики УрО РАН (Екатеринбург).

\section{7. Список литературы}

1. Revishvili A.S., Wissner E., Lebedev D.S., Lemes C., Deiss S., Metzner A., Kalinin V.V., Sopov O.V., Labartkava E.Z., Kalinin A.V., et al. Validation of the mapping accuracy of a novel non-invasive epicardial and endocardial electrophysiology system. Europace. 2015. P. 339.

2. Geuzaine C., Remacle J.-F. Gmsh: A 3-d finite element mesh generator with built-in pre-and postprocessing facilities. International Journal for Numerical Methods in Engineering. 2009. V. 79. № 11. P. 1309-1331.

3. Boulakia M., Cazeau S., Fernández M.A., Gerbeau J.-F., Zemzemi N. Mathematical modeling of electrocardiograms: a numerical study. Annals of biomedical engineering. 2010. V. 38. № 3. P. 1071-1097.

4. Ten Tusscher K.H.W.J., Panfilov A.V. Alternans and spiral breakup in a human ventricular tissue model. American Journal of Physiology-Heart and Circulatory Physiology. 2006. V. 291. № 3. P. H1088-H1100.

5. Bayer J.D., Blake R.C., Plank G., Trayanova N.A. A novel rule-based algorithm for assigning myocardial fiber orientation to computational heart models. Annals of biomedical engineering. 2012. V. 40. № 10. P. 2243-2254.

6. Keller D.U.J., Weiss D.L., Dossel O., Seemann G. Influence of IKs heterogeneities on the genesis of the t-wave: A computational evaluation. IEEE Transactions on Biomedical Engineering. 2012. V. 59. № 2. P. 311-322.

7. Mirams G.R., Arthurs C.J., Bernabeu M.O., Bordas R., Cooper J., Corrias A., Davit Y., Dunn S.-J., Fletcher A.G., Harvey D.G., et al. Chaste: an open source $\mathrm{C}++$ library for computational physiology and biology. PLoS Comput. Biol. 2013. V. 9. № 3. P. e1002970. 